\title{
NONNEGATIVE CURVATURE ON PIECEWISE CONSTANT CURVATURE SPACES
}

\author{
ROBERT PESZEK
}

\begin{abstract}
We consider piecewise flat and piecewise spherical spaces. We prove that every piecewise flat cobordism which is a product near the boundary and has nonnegative curvature must be trivial in the metric sense. We also obtain several restrictions for piecewise $p$-spherical manifolds with nonnegative curvature and diameter equal to $\pi p$. We prove that such a manifold must be homeomorphic to a sphere and that it is a disjoint union of minimal paths connecting two points, which have length $\pi p$.
\end{abstract}

\section{INTRODUCTION}

In this work we consider special metrics on piecewise linear manifolds, which we will call PL-manifolds. We study piecewise flat manifolds consisting of flat simplexes and piecewise spherical manifolds consisting of spherical simplexes. We relate the local geometry of such manifolds to their global topology. The geometric structure which we will study includes cone-like singularities. Structural complications arise only from the combinatorial aspects of the problem.

We define the curvature as in [CMS] and [C]. This definition is traced back to $T$. Regge (see $[R]$ ). Cheeger, Muller, and Schrader showed that it is an extension of scalar curvature to PL-manifolds.

In this work we study piecewise flat and $p$-spherical manifolds which have nonnegative curvature. The assumption of nonnegativity for piecewise flat manifolds happens to be very strong, as strong as nonnegativity of sectional curvature for the Riemannian case (see also [S]). In view of [C] it can be seen that this assumption is even stronger than nonnegativity of sectional curvature. To support these remarks we prove the following in $\S 5$ :

Theorem 5.1. Let $\left(W^{m}, M_{1}, M_{2}\right)$ denote the connected cobordism between manifolds $M_{1}$ and $M_{2}$. Assume that $W$ is a piecewise flat manifold and is isometric to the product $M_{i} \times[0, \varepsilon)$ near the manifolds $M_{1}$ and $M_{2}$. Assume also that $W$ has nonnegative curvature everywhere. Then the manifold $W$ is isometric to the product $M_{1} \times I$.

Received by the editors January 16, 1990 and, in revised form, June 29, 1990.

1991 Mathematics Subject Classification. Primary 53C20, 57Q20, 57Q15, 53C22.

The author expresses his gratitude to M. Lewkowicz, B. Hajduk, and P. Gajer for important stimulation, help, and patience. Further, he thanks R. Rostamian for help in the preparation of this paper. 
An analogous theorem is true for sectional curvature in the smooth case (see Theorem A.1).

Simple observation shows that a piecewise flat manifold $M^{n}$ with positive curvature admits a piecewise spherical metric of positive curvature. Moreover, nonnegativity and positiveness of curvature for piecewise spherical manifolds have the same "strength" since a small perturbation of a $p$-spherical manifold (or piecewise flat manifold with positive curvature) gives a piecewise $(p-\varepsilon)$ spherical (respectively $\varepsilon$-spherical) manifold with positive curvature. Cheeger proved in [C] that a piecewise flat manifold $M^{n}$ with nonnegative curvature is a real homology manifold and that $b^{i}\left(M^{n}\right) \leq\left(\begin{array}{l}n \\ i\end{array}\right)$. A piecewise spherical or piecewise flat manifold with positive curvature is shown to be a real homology sphere.

We prove the following

Corollary 4.3. Let $M^{n}$ be a p-spherical manifold with nonnegative curvature, $p<\infty$. If $\operatorname{diam}\left(M^{n}\right)=\pi p$, then $M^{n}$ is homeomorphic to the standard sphere $S^{n}$.

This corollary may also be considered to be a weak analogue of Toponogov's Maximal Diameter Theorem in Riemannian geometry. As we will explain in Corollary 4.2 the assumption that $\operatorname{diam}\left(M^{n}\right)=\pi p$ may be replaced by $\operatorname{diam}\left(M^{n}\right) \geq \pi p$.

We obtain the following geometric result:

Theorem 1.1. Let $W^{n}$ denote a p-spherical manifold with nonnegative curvature, $p<\infty$. Assume that there exists a pair of points $m_{1}, m_{2} \in W$ such that $\rho\left(m_{1}, m_{2}\right)=\pi \cdot p$. Then for each point $z \in W$ there exists a unique minimal geodesic $\gamma_{z}$ from $m_{1}$ to $m_{2}$ which passes through $z$. In particular, $L\left(\gamma_{z}\right)=\pi p$.

Observe that Theorem 4.1 states that $W^{n} \backslash\left\{m_{1}, m_{2}\right\}$ can be decomposed into a family of disjoint geodesics. This theorem constitutes an important step in the proof of Theorem 5.1 and Corollary 4.3.

Although the above theorems are similar to some theorems in Riemannian geometry, their proofs involve different techniques. The main idea in our proofs is the analysis of suitable families of geodesics.

\section{Preliminaries}

We begin with a brief review of some definitions. We make no distinction in notation between an abstract simplicial complex and its metrical or topological realization such as the metric complex defined below. We consider only finite complexes. We will be especially interested in PL-manifolds (see [RS] for a definition) with suitable, defined below, metrics.

We will indicate when the manifold is noncompact, nonconnected, or with boundary. We use the following notation:

(a) $\operatorname{dim}(a):=$ the dimension of simplicial complex $a$;

(b) $b^{i} \prec a^{j}:=$ simplex $b$ of dimension $i$ is a face of simplex $a$ of dimension $j$;

(c) $b^{i} \prec M^{n}:=b$ is a simplex of dimension $i$ in a complex $M^{n}$;

(d) $S_{r}^{n}:=$ the standard $n$-dimensional sphere of radius $r$; 
(e) $S_{r}^{n+}:=$ the hemisphere of radius $r$, i.e., the set of points $\left(x_{0}, \ldots, x_{n}\right)$ such that $x_{0}^{2}, \ldots, x_{n}^{2}=1$ and $x_{0} \geq 0$;

(f) $S_{\infty}^{n}:=\mathbf{R}^{n}:=$ Euclidean $n$-space;

(g) $S_{\infty}^{n+}:=\mathbf{R}^{n+}:=$ Euclidean; half $n$-space, i.e., the set of points $\left(x_{1}, \ldots, x_{n}\right)$ such that $x_{1} \geq 0$.

If $X, Y \subset \mathbf{R}^{n}$, then the join $X * Y$ is defined to be the union of all straightline segments from $x$ to $y$, where $x$ runs through all points of $X$ and $y$ over all points of $Y$. If $X, Y \subset S_{r}^{n}$ and if no point of $X$ is antipodal to any point of $Y$, then the join $X * Y$ is defined to be the union of the shortest geodesic segments which connect points $x \in X$ and $y \in Y$.

Let $x_{0}, \ldots, x_{n}$ lie in some Euclidean space $\mathbf{R}^{n}$ (or in $S_{r}^{n}$ ). The set $\Delta=$ $x_{0} * \cdots * x_{n}$ is called a linear (or $p$-spherical) simplex if and only if $\operatorname{dim}(\Delta)$ $=n$.

The complex $M$ is metrized by giving, for each simplex $\delta \prec M$, a linear (or $p$-spherical) simplex $\Delta$ in some Euclidean space $R^{n}$ (or sphere $S^{n}$ ) and a homeomorphism $f_{\delta}: \delta \rightarrow \Delta$ such that, whenever $\delta_{1} \prec \delta_{2}$, the induced homeomorphism $\Delta_{1} \rightarrow f_{\delta_{2}}\left(\delta_{1}\right)$ is an isometry (here $\Delta_{1}=f_{\delta_{1}}\left(\delta_{1}\right)$ ).

We call such a complex a linear complex (or $p$-spherical complex). To simplify the notation we will sometimes denote the linear complex as an $\infty$ spherical complex.

The map $f$ between complexes $K_{1}$ and $K_{2}$ is an isometry if and only if there are subdivisions of $K_{1}$ and $K_{2}$ such that $f$ gives a simplicial mapping which is an isometry on each simplex.

Hence a $p$-spherical metric is well defined on a PL-manifold and does not depend of the choice of triangulation.

A path $\alpha:\left[t_{1}, t_{2}\right] \rightarrow K$ is piecewise geodesic if there exists a subdivision $I^{\prime}$ of the interval $I=\left[t_{1}, t_{2}\right]$ such that, for each one-dimensional simplex $\delta \prec I^{\prime}$, $\alpha(\delta)$ is contained in a single simplex of $K$ and $\alpha \mid \delta$ is a geodesic segment in this simplex. The length of such a path will be denoted by $L(\alpha)$.

The distance $\rho_{K}$ on the $p$-spherical complex $K, p \leq \infty$, is defined as follows:

$$
\rho_{K}(x, y)=\inf \{L(\gamma): \gamma \text { is piecewise geodesic path from } x \text { to } y\}
$$

(see [S] for details).

Let $M^{n}$ be a PL-manifold which is a $p$-spherical complex, $p \leq \infty$. We will call such a manifold a piecewise $p$-spherical manifold or simply a $p$-spherical manifold. If $p=\infty$ we will also call $M^{n}$ a piecewise flat manifold.

Let $M^{n-2}$ denote the $n-2$ skeleton of $M$, i.e., the complex consisting of all simplexes with dimension $\leq n-2$. Notice that the set $M^{n} \backslash M^{n-2}$ forms a smooth Riemannian manifold with constant sectional curvature equal to $1 / p$ (we put $1 / \infty=0)$. This is one reason to investigate the contribution to geometry given by singularities "assembled" on the $n-2$ skeleton.

The following is a well-known analogue of the scalar curvature in the case of $p$-spherical manifolds. This definition is due to Regge (cf. [R]). We refer to [CMS] for details. First we introduce the notation $\left[\Delta^{i}, \Delta^{j}\right]$ for the dihedral angle at the face $\Delta^{i}$ of $\Delta^{j}$. Here we mean the unit normals to $\Delta^{i}$ at $x \in$ $\operatorname{int}\left(\Delta^{i}\right)$, which point into $\Delta^{j}$. We use the normalization for which all unit spheres have $(n-1)$-Lebesgue measure 1 . We then set 
(i) $\tau\left(\Delta^{n-2}\right)=1-\sum_{\Delta^{n} \succ \Delta^{n-2}}\left[\Delta^{n-2}, \Delta^{n}\right]$.

We will call this number the defect of the simplex $\Delta^{n-2}$. The contribution to curvature given by the $n-2$ skeleton is measured by the following measure:

(ii) $K(U)=\sum_{\Delta^{n-2} \prec M}\left|\Delta^{n-2} \cap U\right| \cdot \tau\left(\Delta^{n-2}\right)$,

where the symbol $|\cdot|$ denotes the $n-2$ volume.

We will consider a $p$-spherical manifold with nonnegative curvature. This denotes that each defect $\tau\left(\Delta^{n-2}\right)$ is nonnegative.

Although (ii) is considered to be analogous to the scalar curvature, the assumption of nonnegative curvature $K$ gives us strong information about, for example, topology. There are indications that the information obtained from $K \geq 0$ is as strong as that of nonnegativeness of sectional curvature in the smooth case, (see $[\mathrm{S}]$ ), or even stronger (see [C]).

A path $\alpha:\left[t_{1}, t_{2}\right] \rightarrow M$ from $x$ to $y$ is called minimal if it is parametrized by arc-length and if $L(\alpha)=\rho(x, y)=t_{1}-t_{2}$ (compare also the definition given in Remark 4.3). Such a path will be also called a minimal geodesic.

A geodesic is a path $\alpha$ which is locally minimal; that is, $t \in\left[t_{1}, t_{2}\right]$ has a neighborhood $[s, d] \subset\left[t_{1}, t_{2}\right]$ such that $\alpha \mid[s, d]$ is minimal. Note that a geodesic is a piecewise geodesic path (i.e., forms a geodesic segment in each simplex).

Let $\gamma^{-}\left(t_{0}\right)$ and $\gamma^{+}\left(t_{0}\right)$ denote vectors tangent to the curve $t \rightarrow \gamma(t), t \leq t_{0}$, and $t \rightarrow \gamma(t), t \geq t_{0}$, respectively.

We will use the following notation:

The complex $\operatorname{star}(x, M)$ is the complex containing all simplexes including the point $x$ and all their faces.

The complex $\operatorname{link}(x, M)$ is the subcomplex of the complex $\operatorname{star}(x, M)$, consisting of all simplexes not including the point $x$.

Denote the ball of radius $r$ about $x$ as

$$
B(r, x, M)=\{z \in M: \rho(x, z) \leq r\}
$$

and the sphere of radius $r$ about $x$ as

$$
S(r, x, M)=\{z \in M: \rho(x, z)=r\} \text {. }
$$

Notice that if $M^{n}$ is a PL-manifold and $r<\rho(x, \operatorname{link}(x, M))$ then $S(r, x, M)$ is PL-isomorphic to the sphere, and $B(r, x, M)$ is PL-isomorphic to a disc.

We use the symbol $\dot{B}(r, x, M)$ to denote the interior of $B(r, x, M)$; i.e., $\dot{B}(r, x, M)=\{z \in M: \rho(x, z)<r\}$ if $r$ is sufficiently small.

\section{DEFECT SPHERES}

The defects defined in the previous section give us a piece of information which is hidden in the small spheres $S(r, x, M)$, where $r<\rho(x, \operatorname{link}(x, M))$. Therefore these spheres will be called defect spheres.

The following lemma gives us some information about defect spheres. This lemma is due to D. Stone. We refer to [S] for details of the proof. First we explain some symbols: Let us abbreviate $S(r, \bar{p}, M)$ and $B(r, \bar{p}, M)$ 
as $S$ and $B$. Let $M$ denote the $p$-spherical manifold $M^{n}$. Let the mapping

$$
\Psi: \operatorname{star}(\bar{p}, M) \backslash\{\bar{p}\} \rightarrow S
$$

denote the radial projection from $\bar{p}$. Set

$$
q= \begin{cases}p \sin (r / p) & \text { for } p<\infty, \\ r & \text { for } p=\infty\end{cases}
$$

Lemma 3.1. Assume that $r<\frac{1}{2} \cdot \rho(x, \operatorname{link}(x, M))$. Then the following hold for all $x, y \in B$ different from $\bar{p}$ :

(i) $\rho_{M^{n}}(x, y)=\rho_{\text {star }(\bar{p}, M)}(x, y)$.

(ii) The segments $x-\bar{p}-y$ constitute the only minimal path from $x$ to $y$ in $\operatorname{star}(\bar{p}, M)$ if and only if $\rho_{S}(\Psi(x), \Psi(y)) \geq \pi q$.

(iii) If $\rho_{S}(\Psi(x), \Psi(y)) \leq \pi q$ then there exists a bijection $\Psi$ : \{minimal paths from $x$ to $y$ in $B\} \rightarrow\{$ minimal paths from $\Psi(x)$ to $\Psi(y)$ in $S\}$ such that $\operatorname{im} \Psi(\alpha)=\Psi(\operatorname{im}(\alpha))$.

We state only the main idea of the proof. We need the following construction: Let $\alpha$ be the minimal path in $S$ from $\Psi(x)$ to $\Psi(y)$. Consider the join $\alpha * \bar{p}$. This join is isometric as a metric complex with intrinsic metric to a part of a disc $B\left(z, r, S_{p}^{2}\right)$ bounded by two geodesics (parts of great circles) $\gamma_{1}, \gamma_{2}$ starting at point $z$. Observe that the measure of the angle between $\gamma_{1}$ and $\gamma_{2}$ is equal to $L(\alpha) / q$. Sets of the type $\bar{p} * \alpha$ play a crucial role in the proof of Lemma 3.1. Mainly, we can compute the length of the minimal path from $x$ to $y$ and this tells us if it is a minimal path in $B$. Observe that in case $L(\alpha) \leq \pi q$ this minimal path is simply a geodesic segment connecting $x$ and $y$ in $S_{p}^{2}$.

Now we restrict our attention to the case in which $L(\alpha) / q=\pi$. Let $M^{n}$ denote a $p$-spherical manifold with nonnegative curvature. Let $\gamma:\left[t_{1}, t_{2}\right] \rightarrow M$ denote the geodesic. Consider $S(r, \gamma(t), M)$ the defect sphere, where $r$ is sufficiently small, i.e., the following inequalities hold:

$$
r<\frac{1}{2} \rho(x, \operatorname{link}(x, M)) \text { and } t_{1}+r<t<t_{2}-r .
$$

Lemma 3.1 implies that $\rho_{S}(\gamma(t-r), \gamma(t+r))=\pi q$.

Let $\phi$ be a path in $S(r, \gamma(t), M)$ from $\gamma(t-r)$ to $\gamma(t+r)$. Clearly $L(\gamma)=$ $\pi q$. Consider the join $x * \phi$. We will call such a join a page. Now note that from Lemma 3.1 every geodesic segment on a page is geodesic in $M$.

All that has been said gives the following

Corollary 3.1. The join $x * \phi$ is isometric to the half ball $B\left(x, r, S_{p}^{2+}\right)$, where $\left.\gamma\right|_{(t-r, t+r)}$ forms a part of the boundary of $S_{p}^{2+}$. In particular, the path $\phi$ is perpendicular to $\gamma$ (on the page) at both points $\gamma(t-r)$ and $\gamma(t+r)$. Moreover $x * \phi$ forms a totally geodesic surface in each simplex from $\operatorname{star}(x, M)$.

\section{SPHERE THEOREMS}

In this section we prove Theorem 4.1 which gives us useful information about $p$-spherical manifolds, $p<\infty$. In particular, we will be able to explain the structure of defect spheres.

Theorem 4.1. Let $W^{n}$ denote a p-simplicial manifold with nonnegative curvature, $p<\infty$. Assume that there exists a pair of points $m_{1}, m_{2} \in W$ such 
that $\rho\left(m_{1}, m_{2}\right)=\pi \cdot p$. Then for each point $z \in W$ there exists a unique minimal geodesic $\gamma_{z}$ from $m_{1}$ to $m_{2}$ which passes through $z$. In particular, $L\left(\gamma_{z}\right)=\pi p$.

We also have the following

Corollary 4.1. Let $M$ be p-spherical manifold with nonnegative curvature, $p \leq$ $\infty$. Then for every defect sphere $S(r, \bar{p}, M)$, where $r<\frac{1}{2} \rho(x, \operatorname{link}(x, M))$ is as in Lemma 3.1, the following condition holds:

If $\rho(x, y)=\pi q$ for some pair $x, y$, then for an arbitrary point $z$ we have

$$
\rho_{S(r, \tilde{p}, M)}(x, z)+\rho_{S(r, \bar{p}, M)}(y, z)=\pi q
$$

for $q$ as in Lemma 3.1.

Proof of Corollary 4.1. Observe that the sphere $S(r, \bar{p}, M)$ has a $q$-spherical metric with nonnegative curvature. Applying Theorem 4.1, there exists a suitable minimal geodesic $\gamma$ from $x$ to $y$ which passes through $z$. Since the parts of $\gamma$ from $x$ to $z$ and from $z$ to $y$ are the minimal paths, it follows that $\rho_{S(r, \tilde{p}, M)}(x, z)+\rho_{S(r, \bar{p}, M)}(y, z)=\pi q$, which completes the proof.

Note that Corollary 4.1 in dimension $n$ follows from Theorem 4.1 in dimension $n-1$. We prove this theorem using induction on dimensions. It is obvious that Theorem 4.1 holds in dimension 1 (compare the definition of nonnegative curvature in $\S 2$ ). Therefore we assume that Theorem 4.1 holds in dimension $n$.

First we prove a few lemmas:

Lemma 4.1. Let $Z$ and $Y$ denote the closed subsets of the p-spherical manifold $N, p \leq \infty$. Set $\rho(Z, Y)=d$. Assume that $\gamma_{n}$ is the pointwise convergent sequence of minimal geodesics which connects sets $Z$ and $Y$. Assume also that $L\left(\gamma_{n}\right)=d$ for each $n$. We assert that $\lim _{n \mapsto \infty} \gamma_{n}$ is a minimal geodesic and $L(\gamma)=d$.

Proof. Let $0 \leq t_{1} \leq t_{2} \leq \cdots \leq t_{m} \leq d$ be an arbitrary fixed sequence of numbers. Since $\gamma\left(t_{i}\right)=\lim _{n \mapsto \infty} \gamma_{n}\left(t_{i}\right)$, we have:

$$
\rho\left(\gamma\left(t_{i}\right), \gamma\left(t_{i+1}\right)\right)=\lim \rho\left(\gamma_{n}\left(t_{i}\right), \gamma_{n}\left(t_{i+1}\right)\right)=\lim t_{i+1}-t_{i}=t_{i+1}-t_{i}
$$

By definition

$$
L(\gamma)=\lim _{m \rightarrow \infty} \sum_{\left(t_{i}, t_{i+1}\right) \in \eta_{m}} \rho\left(\gamma\left(t_{i}\right), \gamma\left(t_{i+1}\right)\right)
$$

where $\eta_{m}$ denotes the net of intervals included in $[0, d]$. Recall that the curve has a length if and only if this limit exist. In our case,

$$
\begin{aligned}
\lim _{m \rightarrow \infty} & \sum_{\left(t_{i}, t_{i+1}\right) \in \eta_{m}} \rho\left(\gamma\left(t_{i}\right), \gamma\left(t_{i+1}\right)\right) \\
= & \lim _{m \rightarrow \infty} \sum_{\left(t_{i}, t_{i+1}\right) \in \eta_{m}} \lim _{n \rightarrow \infty} \rho\left(\gamma_{n}\left(t_{i}\right), \gamma_{n}\left(t_{i+1}\right)\right) \\
= & \sum_{\left(t_{i}, t_{i+1}\right) \in \eta_{m}} t_{i+1}-t_{i}=d
\end{aligned}
$$

so $L(\gamma)$ exists and is equal to $d$. From this it follows that $\gamma$ is the minimal geodesic connecting sets $X$ and $Y$, and $L(\gamma)=d$. 
Remark 4.1. It is easy to define a geodesic in an arbitrary metric space. Clearly, the path $\gamma$ is a minimal geodesic if and only if $\rho\left(\gamma\left(t_{1}\right), \gamma\left(t_{2}\right)\right)=t_{2}-t_{1}$ for every $0 \leq t_{1} \leq t_{2} \leq d$. Now observe that the proof of Lemma 4.1 is valid under the weaker assumption that $N$ is a metric space.

Let $M^{n}$ be as in Corollary 4.1. We have

Lemma 4.2. The geodesics with fuxed length in $M^{n}$ are uniquely determined by their beginning point and their beginning tangent vector.

Proof. Let $\gamma_{1}, \gamma_{2}:[0, d] \rightarrow M$ have the same beginning point and beginning tangent vector. Set $t_{0}=\inf \left\{t: \gamma_{1}(t) \neq \gamma_{2}(t)\right\}$. Clearly $t_{0}>0$. Consider a sufficiently small defect sphere $S=S\left(r, \gamma_{1}\left(t_{0}\right), M\right)$. We can choose the value of $r$ such that $\operatorname{im}\left(\gamma_{2} \cap S\right)=\{u, y\}$ and $\operatorname{im}\left(\gamma_{1} \cap S\right)=\{u, w\}$, where $w \neq y$. Set $u=m_{1}, w=m_{2}$, and $y=z$. Applying Corollary 4.1 we obtain $\rho_{S}(u, w)+\rho_{S}(w, z)=\pi q$, where

$$
q= \begin{cases}p \sin (r / p) & \text { for } p<\infty, \\ r & \text { for } p=\infty .\end{cases}
$$

But Lemma 3.1 implies that $\rho_{S}(u, w)=\pi q$ and $\rho_{S}(u, z)=\pi q$. Therefore $\rho_{S}(y, w)=0$. Hence $y=w$, which contradicts our assumption.

Proof of Theorem 4.1. As we have pointed out, this theorem is true in dimension 1. We will argue using an induction on dimension. We assume that Corollary 4.1 and Theorem 4.1 are true for all defect spheres in the manifold $W=W^{m}$.

Let $\gamma_{v}$ denote the geodesic from point $m_{1}$ in the manifold $W$ with initial vector $v$. We assume that $\gamma_{v}$ is as long as possible, while still being minimal. The mapping $v \rightarrow \gamma_{v}$ is well defined (see Lemma 4.2). Let $V$ denote the set of all vectors $v$ such that $\gamma_{v}$ is the minimal path from $m_{1}$ to $m_{2}$, and set

$$
W^{\prime}=\bigcup_{v \in V} \operatorname{im}\left(\gamma_{v}\right)
$$

We begin with the proof that the union $\bigcup_{v \in V} \operatorname{im}\left(\gamma_{v}\right)$ is a disjoint union. Suppose that $\gamma_{v}\left(t_{1}\right)=\gamma_{w}\left(t_{2}\right)$ for some $v, w \in V$. Let, for example, $t_{1} \leq t_{2}$. Set

$$
\gamma^{\#}= \begin{cases}\gamma_{v}(t) & \text { for } t \leq t_{1}, \\ \gamma_{w}\left(t+t_{2}-t_{1}\right) & \text { for } t \geq t_{1} .\end{cases}
$$

We have $L\left(\gamma^{\#}\right)=\pi p-t_{2}-t_{1}$. Because $\gamma^{\#}$ is a path from $m_{1}$ to $m_{2}$, we obtain that $t_{1}=t_{2}$ and that $\gamma^{\#}$ is a minimal path. Lemma 4.2 implies that $\gamma^{\#}=\gamma_{v}$ and $\gamma^{\#}=\gamma_{w}$. Hence $v=w$.

Lemma 4.1 and the Arzela-Ascoli Theorem show that $W^{\prime}$ is closed: Let $\left\{z_{n}\right\}_{n \in N}$ denote the sequence of points from $W^{\prime}$. Let also $z_{n} \rightarrow z$. We will prove that $z \in W^{\prime}$. Let $z_{n} \in \operatorname{im}\left(\xi_{n}\right)$, where $\xi_{n}:[0, \pi p] \rightarrow W^{\prime}$ is a minimal path from $m_{1}$ to $m_{2}$. The Arzela-Ascoli Theorem shows that there exist a subsequence $\xi_{n(k)}$ of geodesics which is uniformly convergent to the path $\xi$. Lemma 4.1 implies that $\xi$ is a geodesic minimizing the distance between $m_{1}$ and $m_{2}$. Clearly $z \in \operatorname{im}(\xi)$. Hence $z \in W^{\prime}$, and this completes the proof that $W^{\prime}$ is closed. 
Now we show that $W^{\prime}$ is open. Choose a geodesic $\gamma=\gamma_{v}$, where $v \in V$. We shall construct the geodesic which minimizes the distance between $m_{1}$ and $m_{2}$ in some neighborhood of the geodesic $\gamma$.

There is finite set of numbers $0=t_{1} \leq \cdots \leq t_{k}=\pi p$ and a finite set of balls $B_{i}=B\left(r_{i}, \gamma\left(t_{i}\right), W\right)$ satisfying the conditions of Corollary 3.1 and the inclusion $\operatorname{im}(\gamma) \subset \bigcup_{i=1}^{n} \dot{B}_{i}$. Observe that except for finitely many numbers in $0 \leq t \leq \pi p$ both vectors $\gamma^{-}(t)$ and $\gamma^{+}(t)$ are tangent to the same simplex $\Delta=\Delta(t) \in W$.

Let $t_{i}^{\prime}$ be such a number satisfying the condition $\gamma\left(t_{i}^{\prime}\right) \in B_{i} \cap B_{i+1}$.

We construct the modified orthogonal space at the point $\gamma\left(t_{i}^{\prime}\right)$ : Let $\left\{\Delta_{j}^{n}\right\}$ denote the set of all $n$-dimensional simplexes from $\operatorname{star}\left(\gamma\left(t_{i}^{\prime}\right), W\right)$. We can consider the simplex $\Delta_{j}^{n}$ as a subset of the standard $n$-dimensional sphere $S_{p}^{n}$. We can extend the path $\gamma$ to the geodesic $\gamma^{*}$, which is a great circle in $S_{p}^{n}$. Put the parametrization on $\gamma^{*}$ such that $\gamma^{*}\left(t_{i}^{\prime}+\delta\right)=\gamma\left(t_{i}^{\prime}+\delta\right)$ for sufficiently small values of $\delta$. Let $\varepsilon$ be such that $B\left(\varepsilon, \gamma\left(t_{i}^{\prime}\right), W\right) \subset \dot{B}_{i} \cap \dot{B}_{i+1}$ and $\varepsilon<$ $\rho\left(\gamma\left(t_{i}^{\prime}\right), \operatorname{link}\left(\gamma\left(t_{i}^{\prime}\right)\right)\right)$. Denote

$$
K_{j}^{\prime}=\Delta_{j}^{n} \cap S\left(t_{i}^{\prime}, \gamma^{*}(0), S_{p}^{n}\right) \cap B\left(\varepsilon, \gamma\left(t_{i}^{\prime}\right), W\right) .
$$

Here we identify $B\left(\varepsilon, \gamma\left(t_{i}^{\prime}\right), W\right)$ with the suitable part of $S_{p}^{n}$. We define $K_{i}=$ $\bigcup_{j} K_{j}^{\prime}$.

Let $\gamma\left(t_{i}\right) * \phi^{i}$ be the page (defined in the previous section) in the ball $B_{i}$. According to Corollary 3.1 we can consider this page as a ball in the hemisphere $S_{p}^{2+}$.

Lemma 4.3. Sets $K_{i} \cap\left(\gamma\left(t_{i}\right) * \phi^{i}\right)$ and $K_{i} \cap\left(\gamma\left(t_{i+1}\right) * \phi^{i+1}\right)$ form circles of radius $t_{i}^{\prime}$ perpendicular to the geodesic $\gamma$, i.e., the part of a great circle which is the boundary of $S_{p}^{2+}$; here $t_{i}^{\prime}$ is the radius in the spherical geometry of $S_{p}^{2+}$, not a Euclidean radius.

Proof. Consider, for example, the set $K_{i} \cap\left(\gamma\left(t_{i+1}\right) * \phi^{i+1}\right)$. The inequality $\varepsilon<$ $\rho\left(\gamma\left(t_{i}^{\prime}\right), \operatorname{link}\left(\gamma\left(t_{i}^{\prime}\right)\right)\right)$ implies that $K_{i} \subset \operatorname{star}\left(\gamma\left(t_{i}^{\prime}\right), W\right)$. Note that the triangulation of $\operatorname{star}\left(\left(\gamma\left(t_{i+1}\right), W\right)\right)$ determines in a natural way (by intersections) the triangulation on the sphere $S_{i+1}=S\left(r_{i+1}, \gamma\left(t_{i+1}\right), W\right)$. Observe that the geodesic $\phi^{i+1}$ in $\operatorname{star}\left(\gamma\left(t_{i+1}-r_{i+1}\right), S_{i+1}\right)$ forms a geodesic segment belonging to one simplex from this star. Hence a path $\phi^{i+1}$ forms an arc, a part of a circle of radius $r_{i+1}$ centered in $\gamma\left(t_{i+1}\right)$ in one of the simplexes from $\operatorname{star}\left(\gamma\left(t_{i+1}-r_{i+1}\right), W\right)=$ $\operatorname{star}\left(\gamma\left(t_{i}^{\prime}\right), W\right) \subset \operatorname{star}\left(\gamma\left(t_{i+1}\right), W\right)$ (note that $r_{i+1}$ is a radius in the $p$-spherical geometry, not a Euclidean radius). Therefore $\left(\gamma\left(t_{i+1}\right) * \phi^{i+1}\right) \cap \operatorname{star}\left(\gamma\left(t_{i}^{\prime}\right), W\right)$ forms a part of a $p$-spherical, two-dimensional simplex, which is totally geodesically imbedded into one simplex from $\operatorname{star}\left(\gamma\left(t_{i}^{\prime}\right), W\right)$.

Since $K_{i} \subset B\left(\varepsilon, \gamma\left(t_{i}^{\prime}\right), W\right)$, we have that $K_{i} \cap\left(\phi^{i+1} * \gamma\left(t_{i+1}\right)\right)$ is the circle of radius $t_{i}^{\prime}$ perpendicular to the geodesic $\gamma$.

Let $z \in K_{i}$. We define $\xi_{z}^{i}$ as a geodesic (part of a great circle) in the page $\phi * \gamma\left(t_{i+1}\right)$ perpendicular at $z$ to the circle $K_{i} \cap\left(\phi * \gamma\left(t_{i+1}\right)\right)$. We assume that $\xi_{z}^{i}$ is so long that it connects $K_{i}$ and $K_{i+1}$. Clearly $L\left(\xi_{z}^{i}\right)=t_{i+1}^{\prime}-t_{i}^{\prime}$. According to the inductive assumption, $S_{i+1}=S\left(r_{i+1}, \gamma\left(t_{i+1}\right), W\right)$ is the disjoint union of minimal geodesics from $\gamma\left(t_{i+1}-r_{i+1}\right)$ to $\gamma\left(t_{i+1}+r_{i+1}\right)$. Hence, using paths $\xi_{z}^{i}$ we can fill some neighborhood of the path $\gamma \mid\left(t_{i}^{\prime}, t_{i+1}^{\prime}\right)$. 
Using this construction for each $i$ and joining suitable paths $\xi_{z}^{i}$ together we obtain a family of paths connecting the spheres $S\left(t_{1}^{\prime}, m_{1}, W\right)$ and $S\left(\pi p-t_{k-1}^{\prime}, m_{2}, W\right)$. These paths have length $t_{k-1}^{\prime}-t_{1}^{\prime}$. We can join such paths with the geodesic segments from $m_{1}$ to $S\left(t_{1}^{\prime}, m_{1}, W\right)$ and with the geodesic segments from $m_{2}$ to $S\left(\pi p-t_{k-1}^{\prime}, m_{2}, W\right)$. Therefore we obtain a family of paths from $m_{1}$ to $m_{2}$.

The length of each path in this family is equal to $\pi p$ and this implies that all these paths are geodesics. This family fills some neighborhood of $\gamma$. Hence $W^{\prime}$ is open. Since $W$ is connected, $W^{\prime}=W$. This completes the proof of Theorem 4.1.

Corollary 4.2. Any p-spherical manifold $W^{n}$ with nonnegative curvature satisfies the inequality $\operatorname{diam}\left(W^{n}\right) \leq \pi p$.

Remark. This inequality may be explained in other words. Stone has pointed out (see $[\mathrm{S}]$ ) that for a $p$-spherical manifold with positive curvature even a stronger inequality holds: $\operatorname{diam}\left(W^{n}\right)<\pi p$. Observe that in this case the set $F=W^{n} \backslash W^{n-2}$ forms a Riemannian manifold which has positive constant sectional curvature and has the property that the interior of each geodesic in $W^{n}$ lies in $F$. We make only a small modification when we change the $p$-spherical metric to a $(p-\varepsilon)$-spherical metric (we keep the lengths of the edges fixed). But in this way we obtain a $(p-\varepsilon)$-spherical manifold with positive curvature. Hence Stone's Remark may be used.

Proof of Corollary 4.2. Assume that $x, y \in W^{n}$ satisfies $\rho_{W}(x, y)>\pi p$. Let $\gamma$ be a minimal geodesic from $x$ to $y$. Then let $m_{1}=\gamma(0)=x$ and $m_{2}=\gamma(\pi p)$. It follows from the definition of a minimal geodesic follows that $\rho\left(m_{1}, m_{2}\right)=$ $\pi p$. From Theorem $4.1 y$ lies on $\gamma_{y}$ and

$$
\pi p=L\left(\gamma_{y}\right)=\rho(x, y)+\rho\left(y, m_{2}\right) \geq \rho(x, y)>\pi p,
$$

which is a contradiction.

We also have the following corollary:

Corollary 4.3. Let $M^{n}$ be a p-simplicial manifold with nonnegative curvature. If $\operatorname{diam}\left(M^{n}\right)=\pi p$, then $M^{n}$ is homeomorphic to the standard sphere $S^{n}$.

Remark 4.2. This corollary may be considered a weak version of Toponogov's Maximal Diameter Theorem in Riemannian geometry. It is not true that $M^{n}$ is always isometric to $S_{p}^{n}$.

One can easily construct a two-dimensional counterexample: Consider $S_{p}^{2}$ and two antipodal points $x, y$. Let $\gamma_{1}$ and $\gamma_{2}$ be minimal geodesics from $x$ to $y$. Let $F$ be the set "between" $\gamma_{1}$ and $\gamma_{2}$. Consider the set $S_{p}^{2} \backslash F$ with identification $\gamma_{1}=\gamma_{2}$. This set is in the natural way a two-dimensional $p$ spherical manifold, which satisfies the conditions of Corollary 4.3 and is not isometric to $S_{p}^{2}$.

Proof of Corollary 4.3. We construct a homeomorphism $\Psi: M^{n} \rightarrow S_{p}^{n}$. Let $x, y \in M^{n}$ be such that $\rho(x, y)=\pi p$. Let $x_{0}, y_{0} \in S_{p}^{n}$ be a pair of antipodal points. Consider the sphere $S(\varepsilon, x, M)$, where $\varepsilon<\rho(x, \operatorname{link}(x, M))$, and a PL-isomorphism $\chi: S(\varepsilon, x, M) \rightarrow S^{n}$. Existence of these follows from the definition of PL-manifolds (compare also [RS]). 
Let $v \in S(\varepsilon, x, M)$; we define $\xi_{v}$ to be the only minimal path from $x$ to $y$ which passes through $v$.

Let $z=\gamma_{v}\left(t_{z}\right)$. We define $\Psi(z)=\exp \left(t_{z} \cdot \chi(v)\right)$. To obtain that $\Psi^{-1}$ is a homeomorphism, it suffices to show that $\Psi^{-1}$ is continuous. Therefore it suffices to show that if vectors $v_{n}$ and $t_{n}$ converge to $v$ and $t$, respectively, then $\gamma_{v_{n}}\left(t_{n}\right)$ converges to $\gamma_{v}(t)$. We show that for any subsequence $n(k)$ there exists a subsequence $n(k(l))$ such that $\gamma_{v_{n(k(l))}}\left(t_{n(k(l))}\right) \rightarrow \gamma_{v}(t)$. Consider an arbitrary subsequence $n(k)$. The Arzela-Ascoli Theorem implies that there exists a subsequence $n(k(l))$ for which the sequence $\gamma_{n(k(l))}$ is convergent. Lemma 4.1 implies that $\lim \gamma_{n(k(l))}$ is a minimal geodesic from $m_{1}$ to $m_{2}$. From uniqueness, proved in Theorem 4.1, we know that $\lim \gamma_{n(k(l))}$ is equal to $\gamma_{v}$, therefore $\gamma_{v_{n}(k(l))}\left(t_{n(k(l))}\right) \rightarrow \gamma_{v}\left(t^{\prime}\right)$.

Now observe that $t^{\prime}=\lim \rho\left(m_{1}, \gamma_{v_{n(k(l))}}\left(t_{n(k(l))}\right)\right)=\lim t_{n}$. This observation completes the proof.

\section{THE COBORDISM THEOREM}

The proof of the following theorem is analogous to that of Theorem 4.1. We may think of it as a version of Theorem 4.1 for $p=\infty$.

Theorem 5.1. Let $\left(W^{m}, M_{1}, M_{2}\right)$ denote the connected cobordism between manifolds $M_{1}$ and $M_{2}$. Assume that $W$ is a piecewise flat manifold and is isometric to the product $M_{i} \times[0, \varepsilon)$ near the manifolds $M_{1}$ and $M_{2}$. Assume also that $W$ has nonnegative curvature everywhere. Then the manifold $W$ is isometric to the product $M_{1} \times I$.

Remark 5.1. A version of this theorem for the Riemannian case will be considered in the Appendix.

Proof of Theorem 5.1. Set $\rho_{W}\left(M_{1}, M_{2}\right)=d$. There exists a neighborhood $U$ of the manifold $M_{1}$ which is isometric to a product $M_{1} \times[0, \varepsilon)$. We say that the geodesic $\gamma$ is perpendicular to a manifold if $\gamma$ is given in this neighborhood by the formula $\gamma(t)=(x, t)$.

Let $\gamma_{x}:\left[0, d_{x}\right] \rightarrow W$ denote the geodesic perpendicular to the manifold $M_{1}$ at a point $x$. Hence, and according to Lemma 4.2, we obtain a well-defined family of geodesics $\left\{\gamma_{x}\right\}_{x \in M_{1}}$ (we assume that the interval $\left[0, d_{x}\right]$ is as large as possible).

Let $\gamma$ be a geodesic which minimizes the distance between the manifolds $M_{1}$ and $M_{2}$ (they exist since $M_{1}$ and $M_{2}$ are compact). One easily obtains the following:

Lemma 5.1. The geodesic $\gamma$ is perpendicular to both manifolds $M_{1}$ and $M_{2}$, hence $\gamma=\gamma_{x_{0}}$ for some $x_{0} \in M_{1}$.

Set $V=\left\{x \in M_{1}: \gamma_{x}\right.$ connects manifolds $M_{1}$ and $M_{2}$, and minimizes the distance between them $\}$, and $W^{\prime}=\bigcup_{x \in V} \operatorname{im}\left(\gamma_{x}\right)$. Note that $W^{\prime}$ is the disjoint union of sets $\operatorname{im}\left(\gamma_{x}\right)$. To see this, suppose a priori that $\gamma_{x}\left(t_{1}\right)=\gamma_{y}\left(t_{2}\right)$ for some points $x, y \in V$. Consider, for example, the case $t_{1} \leq t_{2}$. Set

$$
\gamma^{\#}= \begin{cases}\gamma_{x} & \text { for } t \leq t_{1}, \\ \gamma_{y}\left(t+t_{2}-t_{1}\right) & \text { for } t \geq t_{1} .\end{cases}
$$


The length of this path is equal to $d-\left(t_{2}-t_{1}\right)$. We have $\gamma^{\#}(0) \in M_{1}$ and $\gamma^{\#}(d) \in M_{2}$. This means that $t_{1}=t_{2}$ and $L\left(\gamma^{\#}\right)=d$. Hence $\gamma^{\#}$ is geodesic. According to Lemma 4.2 we obtain $\gamma^{\#}=\gamma_{y}$ and $\gamma^{\#}=\gamma_{x}$. In particular, $x=y$.

As in the proof of Theorem 4.1 we assert that $W^{\prime}$ is both open and closed, therefore $W^{\prime}=W$.

The proof of the fact that $W^{\prime}$ is closed is completely analogous to a suitable part in the proof of Theorem 4.1.

To prove that $W^{\prime}$ is open we choose a geodesic $\gamma=\gamma_{x}$, where $x \in V$. We will construct a family of geodesics in a neighborhood of $\operatorname{im}(\gamma)$. As in Theorem 4.1 we consider a finite family of sufficiently small (as in Lemma 3.1) balls: $B_{i}=B\left(r_{i}, \gamma\left(t_{i}\right), W\right)$, where $0=t_{1} \leq \cdots \leq t_{k}=d$.

We assume that these balls cover the geodesic $\gamma$ (i.e., $\operatorname{im}(\gamma) \subset \bigcup_{i=1}^{n} \dot{B}_{i}$ ). As in the previous theorem, observe that except for finitely many numbers in $0 \leq$ $t \leq d$ both vectors $\gamma^{-}(t)$ and $\gamma^{+}(t)$ are tangent to the same simplex $\Delta=\Delta(t)$. For such a number $t$ we can easily construct an orthogonal complement $\gamma^{\perp}$. We form this set from all complements orthogonal to $\gamma$ in suitable simplexes (i.e., including $\gamma(t))$ in $\operatorname{star}\left(\gamma\left(t_{0}\right), W\right)$. We choose such a number $t_{i}^{\prime}$ in each section $\dot{B}_{i} \cap \dot{B}_{i+1}$. Let $\varepsilon$ be chosen such that $B\left(\varepsilon, \gamma\left(t_{i}^{\prime}\right), W\right) \subset \dot{B}_{i} \cap \dot{B}_{i+1}$ and $\varepsilon<\rho\left(\gamma\left(t_{i}^{\prime}\right), \operatorname{link}\left(\gamma\left(t_{i}^{\prime}\right)\right)\right)$. We define $K_{i}=\gamma^{\perp} \cap B\left(\varepsilon, \gamma\left(t_{i}^{\prime}\right), W\right)$.

It is obvious (see the proof of Theorem 4.1 and Lemma 4.3) that for each page $\gamma\left(t_{i}\right) * \phi$ the set $K_{i} \cap\left(\gamma\left(t_{i}\right) * \phi\right)$ forms an interval perpendicular (on the page) to $\gamma$.

We consider the families of paths, lying on pages, connecting sets $K_{i}$ and $K_{i+1}$ and parallel (on pages) to $\gamma$. By connecting such families of paths we construct curves connecting manifolds $M_{1}$ and $M_{2}$. According to Theorem 4.1 these paths fill some neighborhood of the geodesic $\gamma$. We can easily compute lengths of such paths. They are equal to $d$. This completes the assertion that $W^{\prime}$ is open.

To close the proof of the theorem we should explain that the mapping

$$
F: M_{1} \times I \rightarrow W
$$

given by $F(x, y)=\gamma_{x}(t)$ is an isometry.

Let $\gamma_{x_{0}}$ be a fixed geodesic. According to the construction there exists an $\varepsilon=\varepsilon_{x_{0}}$ such that

$$
B\left(\varepsilon, \gamma_{x_{0}}, W\right)=\bigcup\left\{\gamma_{y}: y \in M_{1}, \rho_{M_{1}}\left(x_{0}, y\right) \leq \varepsilon\right\}
$$

$\left(B\left(\varepsilon, \gamma_{x_{0}}, W\right)\right.$ denotes the set of points $z$ which fulfill $\left.\rho_{w}\left(\operatorname{im}\left(\gamma_{y}\right), z\right) \leq \varepsilon\right)$ and for every point $y_{0} \in B\left(\varepsilon, \gamma_{x_{0}}, W\right)$ we have

(A) $\rho_{W}\left(\gamma_{y_{0}}(t), \gamma_{x_{0}}(t)\right)=\rho_{M_{1}}\left(y_{0}, x_{0}\right)=\operatorname{const}(t)$.

Now observe that the distance on a piecewise flat or piecewise spherical manifold may be defined as $\rho(x, y)=\inf \left\{\int|\dot{\gamma}| d t: \gamma\right.$ is a piecewise geodesic path connecting points $x$ and $y$ \}. Therefore, and since the volume $|\dot{\gamma}|$ is locally defined, it suffices to restrict our attention to any neighborhood $U$ of the point $x \in W$, and to show that the following condition holds:

(B) For each point $x \in W$ there exists a neighborhood containing $x$ such that

$$
\rho_{M_{1} \times I}\left(F^{-1}(x), F^{-1}(y)\right)=\rho_{W}(x, y)
$$

for every point $y$ belonging to $U$.

Choose a point $x \in W$. Let $x=\gamma_{x_{0}}\left(t_{1}\right)$. 
Consider $B(r, x, W)$, where $r \leq \min \left\{\frac{1}{2} \rho(x, \operatorname{link}(x)), \varepsilon\right\}$. Assume that $y \in \operatorname{im}\left(\gamma_{y_{0}}\right)$. Let $\Psi$ denote (as in Lemma 3.1) the radial projection onto $S(r, x, W)$. Hence the point $\Psi(y)$ determines a page $\phi_{\Psi(y)} * \gamma_{x_{0}}\left(t_{1}\right)$ in the ball $B(r, x, W)$ (see Theorem 4.1).

It suffices to show that

(C) $\left(\rho_{W}(x, y)\right)^{2}=\left(\rho_{M_{1}}\left(x_{0}, y_{0}\right)\right)^{2}+\left(t_{1}-t_{2}\right)^{2}$.

The method of construction shows that the segment $\gamma_{x_{0}}\left(t_{2}\right)-\gamma_{y_{0}}\left(t_{2}\right)$ is perpendicular (on the page) to the geodesic $\gamma_{x_{0}}$. From Lemma 3.1 and Corollary 3.1 the distance in $W$ equals the distance on the page. Hence

$$
\left(\rho_{W}(x, y)\right)^{2}=\left(\rho_{W}\left(\gamma_{x_{0}}\left(t_{2}\right), \gamma_{y_{0}}\left(t_{2}\right)\right)\right)^{2}+\left(\rho_{W}\left(\gamma_{x_{0}}\left(t_{2}\right), \gamma_{x_{0}}\left(t_{1}\right)\right)\right)^{2} .
$$

According to (A) we obtain

$$
\left(\rho_{W}\left(\gamma_{x_{0}}\left(t_{2}\right), \gamma_{y_{0}}\left(t_{2}\right)\right)\right)^{2}=\left(\rho_{M_{1}}\left(x_{0}, y_{0}\right)\right)^{2} .
$$

Since the geodesic $\gamma$ is parametrized by the distance between suitable points, we have

$$
\left(\rho_{W}\left(\gamma_{x_{0}}\left(t_{1}\right), \gamma_{x_{0}}\left(t_{2}\right)\right)\right)^{2}=\left(t_{1}-t_{2}\right)^{2} .
$$

This proves $(\mathrm{C})$. Therefore $(\mathrm{B})$ holds. This completes the proof of Theorem 5.1 .

\section{APPENDIX}

As we said already, there is an analogue of Theorem 5.1 in the Riemannian geometry. In this appendix we prove the following theorem, which seems to be well known, but we have not seen it in the literature.

Theorem A.1. Let $\left(W^{m}, M_{1}, M_{2}\right)$ denote the connected cobordism between manifolds $M_{1}$ and $M_{2}$. Assume that $W$ is the Riemannian manifold and is isometric to a product $M_{i} \times[0, \varepsilon)$ near the manifolds $M_{1}$ and $M_{2}$. Assume also that $W$ has nonnegative sectional curvature everywhere. Then the manifold $W$ is isometric to the product $M_{1} \times I$.

This theorem easily follows from the following (see [CE] for a proof):

Theorem A.2 (Cheeger, Gromoll, Toponogov). Let $X$ be a complete (not necessarily compact but without boundary) manifold with nonnegative sectional curvature. Then $X$ may be uniquely described as an isometric product $X^{\prime} \times \mathbf{R}^{k}$, where $X^{\prime}$ is a manifold without boundary which does not include any lines and $\mathbf{R}^{k}$ has standard metric.

Remark A.1. The geodesic $\gamma:(-\infty, \infty) \rightarrow X$ is said to be a line if and only if $\rho\left(\gamma\left(t_{1}\right), \gamma\left(t_{2}\right)\right)=t_{2}-t_{1}$ for each $-\infty<t_{1} \leq t_{2}<\infty$.

Proof of Theorem A.1. Let $\gamma:[0, d] \rightarrow W$ be the geodesic which minimizes the distance between the manifolds $M_{1}$ and $M_{2}$. Let $L(\gamma)=\rho\left(M_{1}, M_{2}\right)=d$. Observe that $\gamma$ is perpendicular to both manifolds $M_{1}$ and $M_{2}$. We identify in a natural way the boundaries of Riemannian manifolds: 


$$
M_{1} \times(-\infty, 0], \quad W, \quad M_{2} \times[d, \infty)
$$

so that we obtain a manifold $X$ which fulfills the conditions of Theorem A.2. We abbreviate $M_{1} \times\{0\}$ as $M_{1}$ and $M_{2} \times\{d\}$ as $M_{2}$.

Set

$$
\gamma^{\#}(t)= \begin{cases}(\gamma(0), t) \in M_{1} \times(-\infty, 0] & \text { if } t \leq 0, \\ \gamma(t) \in W & \text { if } 0 \leq t \leq d \\ (\gamma(d), t) \in M_{2} \times[d, \infty) & \text { if } t \geq d\end{cases}
$$

The path $\gamma^{\#}$ is a line.

It follows from Theorem A.2 that $X=X^{\prime} \times \gamma^{\#} \cong X^{\prime} \times R$. We may assume that the manifold $X^{\prime}$ is such that $X^{\prime} \times\{0\} \cap M_{1} \neq \varnothing$. The manifold $X^{\prime} \times\{0\}$ is totally convex in $X$ (i.e., any geodesic which cuts $X^{\prime} \times\{0\}$ in two points must be included in $\left.X^{\prime} \times\{0\}\right)$. Hence $X^{\prime} \times\{0\} \cap M_{1}$ is totally convex in the manifold $M_{1}$. Since $M_{1}$ is compact, $M_{1} \subset X^{\prime} \times\{0\}$ or $M_{1}=X^{\prime} \times\{0\}$. Therefore, as $X$ is connected, we obtain that $X^{\prime}$ is connected. It follows that $M_{1}=X^{\prime}$. Similarly $X^{\prime} \times\{d\}=M_{2}$. This implies that $W=X^{\prime} \times[0, d]=$ $M_{1} \times[0, d]$.

\section{REFERENCES}

[C] J. Cheeger, A vanishing theorem for piecewise constant curvature spaces, Lecture Notes in Math., vol. 1201, Springer, Berlin and New York, 1985, pp. 35-40.

[CE] J. Cheeger and D. G. Ebin, Comparison theorems in Riemannian geometry, North-Holland, Amsterdam, 1975.

[CMS] J. Cheeger, W. Muller, and R. Schrader, On the curvature of piecewise flat spaces, Comm. Math. Phys. 92 (1984), 405-545.

[R] T. Regge, General relativity without coordinates, Nuovo Cimento 19 (1961), 558-571.

[RS] C. P. Rourke and B. J. Sanderson, Introduction to piecewise-linear topology, Springer, Berlin, Heidelberg, and New York, 1972.

[S] D. Stone, Geodesics in piecewise-linear manifolds, Trans. Amer. Math. Soc. 215 (1976), $1-40$.

Department of Mathematics and Statistics, University of Maryland Baltimore County, Baltimore, Maryland 21228 